\title{
Desenvolvimento do Pensamento Computacional e discussões sobre representação feminina na Computação: um estudo de caso
}

\author{
Andreia Pinheiro dos Santos Silva', João Silvestre dos Santos Franco', \\ Jorge Costa Leite Junior ${ }^{1}$ \\ ${ }^{1}$ Instituto Federal de Educação, Ciência e Tecnologia da Bahia (IFBA) \\ Campus Santo Amaro \\ CEP: 44.200-000 - Santo Amaro - BA - Brasil \\ andreiapinheiro.ifba@gmail.com,\{joao.franco,leitejr\}@ifba.edu.br
}

\begin{abstract}
The difficulties in learning programming and the masculinization of the computing area, are recurrent themes in the researches of this area. This paper exposes a study case which was executed with female students from public schools and incoming students for a technician course in information Technology. This initiative intends to approximate specific knowladges of computing from the students' daily life, through activities supported by Hour of Code and App inventor and Unplugged Science methodology. It will be presented the target audience, the methodological steps, a synthesis of the activities and the results, starting from the experiences of the students and the executor team of the project.
\end{abstract}

Resumo. As dificuldades na aprendizagem de programação e a masculinização da área da Computação são temas recorrentes em trabalhos da área. Este trabalho apresenta um estudo de caso realizado com estudantes do sexo feminino de escolas públicas e ingressantes de um curso técnico em Informática. Esta iniciativa objetiva mediar a construção de diálogos entre os conhecimentos específicos de Computação e o cotidiano dos estudantes, por intermédio de atividades apoiadas nos ambientes Hora do Código e App Inventor e na metodologia da Computação Desplugada. Serão apresentados público-alvo, etapas metodológicas, uma síntese das atividades e resultados, a partir das vivências dos estudantes e da equipe executora do projeto.

\section{Introdução}

No atual contexto da sociedade, as novas tecnologias de comunicação e informação (NTIC) são fundamentais ao desenvolvimento de novas ideias, práticas e metodologias. Compreender as possibilidades de utilização dos recursos tecnológicos torna-se de extrema importância. Para Pinto (2004), "nota-se, nos últimos anos em especial, mudanças em vários setores da sociedade impulsionadas pelo desenvolvimento da tecnologia" [PINTO, 2004]. Nesta perspectiva, a escola, enquanto espaço formador do sujeito, necessita acompanhar as mudanças da sociedade, reestruturando os métodos de ensino e propiciando a aquisição e desenvolvimento de novos saberes relacionados aos processos de ensino e aprendizagem, por intermédio dos avanços tecnológicos. 
VI Congresso Brasileiro de Informática na Educação (CBIE 2017)

Anais dos Workshops do VI Congresso Brasileiro de Informática na Educação (WCBIE 2017)

A aplicação inteligente do computador na educação é aquela que sugere mudanças na abordagem pedagógica, encaminhando os sujeitos para atividades mais criativas, críticas e de construção conjunta. [...] É importante criar um ambiente de ensino e aprendizagem instigante, que proporcione oportunidades para que seus alunos pesquisem e participem na comunidade, com autonomia [FARIA, 2004].

A sociedade reflete desigualdades que, atreladas à questão racial, gênero e sexualidade, geralmente, resultam na falta de oportunidades. Tais reflexos estão presentes na academia e no mundo do trabalho, espaços são evidenciados pelas diferenças de salários e oportunidades em cargos de liderança. Embora haja gradual aumento na inserção de mulheres nas instituições de ensino técnico e superior, esta não se equipara ao contingente masculino presente nestes locais, especialmente quando se trata do ensino tecnológico. Segundo dados do último Censo do Instituto Brasileiro de Geografia e Estatísticas (IBGE), cerca de 78\% dos ingressos nos cursos de Computação são estudantes do sexo masculino e apenas $22 \%$ são do sexo feminino [MACHADO, 2016]. Essa disparidade de gênero é notória em cursos técnicos e de graduação na área da Computação e outras tecnologias, e gera reflexos no mundo do trabalho, sobretudo, no número de profissionais formados na área.

A educação essencialmente voltada para o profissional configura-se como instrumento de reprodução das desigualdades, inclusive de gênero. Alguns autores corroboram esta perspectiva e afirmam que a predominância masculina na área de ciências exatas é, por vezes, resultado de fatores sociais e estruturais como: educação informal e formal, mídias e cultura popular, que influenciam significativamente nas escolhas das meninas para prosseguir na carreira da Computação [ASHCRAFT, 2012]. Neste sentido, estruturar iniciativas que estimulem a inserção do público feminino na área da Computação fortalece a equidade de gênero, desconstruindo paradigmas e minimizando as demandas de mercado, posto que a Ciência da Computação, enquanto mercado em expansão, se destaca como um dos atuais pilares econômicos da sociedade, exigindo uma gama cada vez maior de profissionais [ASHCRAFT, 2012].

Diversos estudos apontam para a necessidade da promoção de iniciativas que tornem os estudantes sujeitos aptos a compreender e participar da dinâmica de construção de tecnologias; dentre as vertentes, destaca-se a programação. Países como os Estados Unidos, já adotaram a programação de Computadores nas séries iniciais, considerando as contribuições da área de Computação ao processo de aprendizagem. Neste sentido, diversas metodologias vêm sendo empregadas com intuito de aproximar crianças e adolescentes com a área tecnológica por intermédio da aprendizagem de programação associada a ferramentas, ambientes de aprendizagem e metodologias, como Scratch [Bezerra \& Dias, 2014], Lightbot [Cassenote \& Antoniazzi, 2015] e Computação Desplugada [BELL et al, 2011]. Estes destacam-se por associar elementos lúdicos ao ensino de programação, permitindo que pessoas de diferentes idades experimentem o ato de programar. Desse modo, a promoção de iniciativas que desmistificam a ideia de que a programação é algo restrito e específico para estudiosos da área de Computação, reforçando a ideia de que o ato de programar é uma habilidade inerente ao mundo contemporâneo, considerando as contribuições dos avanços 
VI Congresso Brasileiro de Informática na Educação (CBIE 2017)

Anais dos Workshops do VI Congresso Brasileiro de Informática na Educação (WCBIE 2017)

tecnológicos para o desenvolvimento da sociedade. Sobre o Pensamento Computacional, sobretudo, cabe destacar que

Pensamento Computacional é uma habilidade fundamental para todos, não somente para cientistas da computação. À leitura, escrita e aritmética, deveríamos incluir Pensamento Computacional na habilidade analítica de todas as crianças. (...) envolve a resolução de problemas, projeção de sistemas, e compreensão do comportamento humano, através da extração de conceitos fundamentais da ciência da computação. O Pensamento Computacional inclui uma série de ferramentas mentais que refletem a vastidão do campo da ciência da computação [WING, 2016].

Neste sentido, este artigo é resultado da experiência de estudantes do curso de Licenciatura em Computação em um projeto de extensão, cuja principal objetivo foi disseminar conhecimentos sobre a Computação a estudantes da rede pública na faixa etária de 11 a 18 anos, principalmente o público feminino. As propostas de ação foram articuladas para despertar a curiosidade acerca das possibilidades de uso das tecnologias, ao trabalhar conceitos basilares de programação, evidenciando as diferentes vertentes da área. Deste modo, buscou-se promover o desenvolvimento do raciocínio lógico, Pensamento Computacional e abstração para resolução de problemas. Suscitadas as discussões sobre a masculinização das ciências exatas e suas tecnologias, sobretudo, da Computação, foi traçado um caminho para superação da instrumentalização do ambiente educativo como espaço de mera formação de mão de obra, numa perspectiva de educação transformadora, além de possibilitar não apenas o consumo da tecnologia, mas a participação dos sujeitos no processo de desenvolvimento tecnológico e na construção do Pensamento Computacional.

Para realizar as devidas considerações sobre a problemática acima apresentada, o presente trabalho foi organizado da seguinte forma: na seção 2, serão apresentados brevemente alguns trabalhos correlatos; na seção 3 , as etapas de planejamento e execução do trabalho, além dos instrumentos de avaliação utilizados no decorrer do projeto, bem como a metodologia empregada. A seção 4 apresenta os resultados e a discussão sobre os dados obtidos com a pesquisa subsidiada pelos ambientes Hora do Código e App Inventor. Por fim, são tecidas as considerações finais.

\section{Trabalhos relacionados}

Dantas e Costa [2013] apresentam uma proposta de ensino de programação com o intermédio do ambiente Hora do Código. Como objetivo principal da pesquisa, os autores apresentam a necessidade de compreender de que forma as habilidades que se apresentam no aprendizado de programação podem ser relacionadas a habilidades inerentes a construção do conhecimento dos alunos. Como resultados, são apresentadas possibilidades que o ambiente oferece, relacionadas ao desenvolvimento de habilidades como o raciocínio lógico e resolução de problemas que, atreladas ao processo de programar, corroboram o enriquecimento do conhecimento dos indivíduos.

Ainda nesta perspectiva, Cavalcante e Costa [2016] trazem contribuições voltadas às competências utilizadas em um curso de programação, que propiciam o desenvolvimento do Pensamento Computacional. Como elemento de investigação, os autores utilizam um framework responsável por avaliar a eficácia de ambientes de 
programação em blocos no desenvolvimento do Pensamento Computacional. Nos resultados obtidos, os autores apresentam a eficiência do framework e as práticas elencadas no ambiente relacionadas ao Pensamento Computacional não reconhecidas pelo framework. Por fim, os autores propõem a análise de outros cursos e outros frameworks relacionados ao Pensamento Computacional.

França e Tedesco [2015] corroboram os ideais de Cavalcante e Costa quando discutem os desafios que circundam o ensino do Pensamento Computacional na educação básica brasileira; evidenciam diferentes pesquisas na área da Computação sobre o Pensamento Computacional, além de apresentar abordagens que possibilitam a utilização da programação enquanto meio de desenvolver o Pensamento Computacional no ensino médio, trazendo como referencial pesquisas desenvolvidas em diferentes estados do Brasil.

Para Gomes e Melo [2013], as tecnologias atreladas ao ensino de programação tornam-se importantes ferramentas para que se constituam experiências de aprendizagem significativas e inovadoras. Assim, os autores elencam procedimentos para melhor aproveitamento do potencial educativo dos dispositivos móveis, através do ambiente App Inventor. Como resultado da investigação, os autores afirmam que no decorrer do processo de construção das aplicações, além de exercitarem a lógica de programação, os estudantes estabelecem conexões entre os conceitos aprendidos e seu cotidiano, aprimorando sua percepção e autonomia.

Em outro trabalho, Gomes e Melo [2013] utilizam o App Inventor no desenvolvimento de um minicurso realizado por intermédio das técnicas de blended learning, cuja proposta tem como objetivo minimizar as dificuldades comumente vistas na aprendizagem de programação, através da apresentação contextualizada dos conteúdos de lógica de programação. Como resultado, os autores apresentam o percentual de aproveitamento da atividade por intermédio da visão dos estudantes, como também a atenuação de dificuldades relacionadas à aprendizagem de programação, como leitura e interpretação de textos, matemática básica e idiomas da ferramenta.

\section{Materiais e métodos}

Nesta seção, são apresentados os passos e os materiais utilizados, bem como os procedimentos planejados desde o início do projeto, para obtenção dos resultados que serão apresentados na seção seguinte.

\subsection{Método de Pesquisa}

Para a coleta e análise dos dados, foi adotada a metodologia qualitativa. Segundo Martins, "As metodologias qualitativas privilegiam, de modo geral, a análise de microprocessos, através do estudo das ações sociais individuais e grupais" [MARTINS, 2004]. Atrelada a metodologia qualitativa, foi utilizada para a concepção da proposta a abordagem do estudo de caso. O Estudo de caso possui como característica a análise de um fenômeno, com intuito de adquirir informações relevantes sobre a classe a que esse fenômeno pertence. Assim,

Estudos de caso são uma estratégia de investigação em que o pesquisador explora profundamente um programa, um evento, uma atividade, um processo ou um ou mais indivíduos. Os casos são 
VI Congresso Brasileiro de Informática na Educação (CBIE 2017)

Anais dos Workshops do VI Congresso Brasileiro de Informática na Educação (WCBIE 2017)

relacionados pelo tempo e pela atividade, e os pesquisadores coletam informações detalhadas usando vários procedimentos de coleta de dados durante um período de tempo prolongado [Creswell, 2010]

Dado o caráter das questões que se apresentam na pesquisa, a abordagem de estudo de caso se adéqua a proposta aqui apresentada, pois permite uma análise aprofundada e detalhada do processo de ensino e aprendizagem de programação.

As intervenções foram realizadas durante dois dias semanais, no turno vespertino, com duração total de 2 horas por aula; no decorrer das intervenções foram utilizados, enquanto instrumentos de coleta de dados questionários pré e pós intervenção, com o objetivo de identificar mudanças na percepção dos estudantes em torno da área da Computação; diário de bordo - a fim de identificar a visão dos professores sobre as atividades realizadas, constatando momentos de dificuldades, pontos positivos, erros e possíveis mudanças a serem realizadas; e atividades diagnósticas - com intuito de observar o desenvolvimento dos participantes, além de identificar possíveis rupturas na compreensão de conceitos e realização de técnicas inerentes a programação em blocos.

\subsection{Plano de Trabalho}

As atividades foram idealizadas a partir da análise do perfil de estudantes, realizada por intermédio de uma ficha de inscrição disponibilizada no período de divulgação nas instituições de ensino da cidade de Santo Amaro, tendo como público alvo estudantes do $8^{\circ}$ e $9^{\circ}$ ano, sobretudo, do sexo feminino. Em relação aos calouros do curso técnico em Informática da instituição onde o projeto foi executado, a etapa de divulgação consistiu em ligações telefônicas e no envio de e-mails, com intuito de apresentar o projeto e angariar a atenção dos futuros estudantes. Após essa etapa, ocorreu o planejamento das ações, considerando o perfil dos 25 estudantes inscritos com faixa etária entre 14 a 15 anos.

O planejamento da ação contemplou a execução de dois blocos de atividades, que juntos totalizaram uma carga horária de 60 horas. O primeiro bloco buscou estimular e desenvolver habilidades de lógica de programação e o Pensamento Computacional, como também apresentar conceitos introdutórios de programação, por intermédio de atividades subsidiadas pela metodologia da Computação Desplugada e o ambiente Hora do Código.

Desenvolvido pela instituição sem fins lucrativos Code.org (http://www.code.org), o Hora do Código é um ambiente virtual cujo intuito é permitir que professores, estudantes e pais, tenham o primeiro contato com o mundo da programação de forma lúdica e eficaz, desmistificando a ideia de que programar é algo difícil. Além de viabilizar o ensino de programação, as atividades disponíveis no ambiente apresentam intervenções subsidiadas pela técnica da Computação Desplugada - "atividades desenvolvidas com o objetivo de ensinar os fundamentos da Ciência da Computação sem a necessidade de computadores" [BELL et al, 2011], visando o desenvolvimento de habilidades como o Pensamento Computacional, raciocínio lógico e abstração. 
O segundo bloco buscou utilizar os conceitos trabalhados nas atividades da fase anterior para construção de aplicações, com o uso da ferramenta App Inventor. Nesta etapa, os estudantes assumiram a responsabilidade na confecção de aplicações de maneira autônoma, na escolha da interface, funcionalidade e objetivos. A finalidade deste bloco foi promover o estímulo da autonomia e evidenciar as potencialidades de utilização dos recursos tecnológicos.

Desenvolvido pelo MIT e atualmente gerenciado pela empresa Google, a ferramenta baseada na linguagem de blocos possibilita a criação de aplicações da mais simples a mais complexa por qualquer pessoa. A ferramenta é disponibilizada gratuitamente e tem como objetivo principal democratizar o desenvolvimento de software, capacitando todas as pessoas, especialmente os jovens, para se transporem de consumidores de tecnologia para se tornarem criadores [MIT App Inventor, 2017].

A escolha do Hora do Código e do App Inventor, enquanto ferramentas de auxílio às atividades, foi resultado da pesquisa bibliográfica realizada, tendo em vista que estas ferramentas, junto com Scratch e Lightbot, se destacavam dentre as mais utilizadas, quando se trata do desenvolvimento de atividades voltadas ao ensino de programação de forma lúdica e apoio ao desenvolvimento de lógica matemática. Além disso, possuem contextos semelhantes e utilizam a mesma linguagem, fatores preponderantes para a escolha das ferramentas.

\section{Resultados e discussão}

Após a realização das etapas elencadas nas seções anteriores e com a aplicação dos questionários pré e pós-intervenção, foram obtidos os resultados, sobre os quais foi possível tecer algumas considerações, conforme exposto nesta seção.

Na primeira atividade prática utilizou-se o ambiente Code.org, como subsídio a alguns conceitos apresentados relacionados à estrutura sequencial, com um quantitativo de 42 pessoas presentes. Este foi o momento mais difícil deste ciclo inicial de atividades do projeto, visto que a estrutura do laboratório não foi capaz de comportar tamanha quantidade de participantes, exacerbadamente superior à estimativa. Na tentativa de otimizar o uso das máquinas e possibilitar a participação de todos os presentes, estes foram separados em pares. Ficou evidente, naquele momento, que seria necessário limitar o número de participantes e o acesso ao laboratório por membros externos, ouvintes não-participantes.

Buscando solucionar o problema de aprendizado provocado pela problemática do excesso de participantes, traçou-se uma estratégia diferenciada, baseada na Computação Desplugada. Observou-se que os estudantes assimilaram de forma muito mais eficiente o conteúdo, desenvolvendo uma reflexão mais profunda sobre os temas tratados. O trabalho se deu, neste momento, no sentido de confrontamento do conteúdo exposto com as dinâmicas realizadas em momentos precedentes e a construção do jogo Flappy Bird. Foram apresentados o conceito de variáveis e uma sucinta introdução sobre o funcionamento do computador, ao mencionar memória RAM e processamento. A transição de estrutura sequencial para estrutura de repetição foi feita de forma suave, de modo que a maior dificuldade apresentada foi na atividade do labirinto, cuja resolução se deu através do uso de comandos fíctícios sequenciais. Neste ponto, os 
VI Congresso Brasileiro de Informática na Educação (CBIE 2017)

Anais dos Workshops do VI Congresso Brasileiro de Informática na Educação (WCBIE 2017)

estudantes apresentaram grande dificuldade em seguir uma sequência eficaz na resolução do labirinto.

A aplicação dos questionários possibilitou a investigação de impressões, críticas e sugestões dos estudantes frente às atividades desenvolvidas no projeto; os questionários continham espaços para identificação com nome e idade, somadas às questões voltadas a área da Computação. Indagados sobre a participação em aulas de programação, todos os estudantes afirmaram não ter participado de intervenções anteriores, porém, um estudante relatou possuir conhecimentos relacionados a linguagem de programação $C$ como resposta à questão sobre utilização de linguagens de programação, se já havia utilizado e qual linguagem utilizou. Entre as opções, estavam as linguagens: Python, Java, C e "de blocos".

Ainda no questionário pré-intervenção, foram apresentadas afirmações onde os estudantes deveriam marcar aquelas relacionadas a sua percepção sobre a área da Computação; as afirmações estavam relacionadas a conhecimentos sobre a profissão de programador, visualizar uma carreira na área da Computação, além de ver facilidades na área. Como resultado dessas afirmações, cerca de 40\% dos estudantes demonstraram não saber quais são as atribuições de um programador; apesar disso, 39\% concordaram que a programação de computadores é algo fácil. Em relação a atuar na área da Computação, os estudantes se mostraram divididos, visto que 36\% mostraram-se neutros, ao passo que 32\% concordaram que a Computação é uma opção considerada, enquanto outros $28 \%$ não pretendem seguir na área.

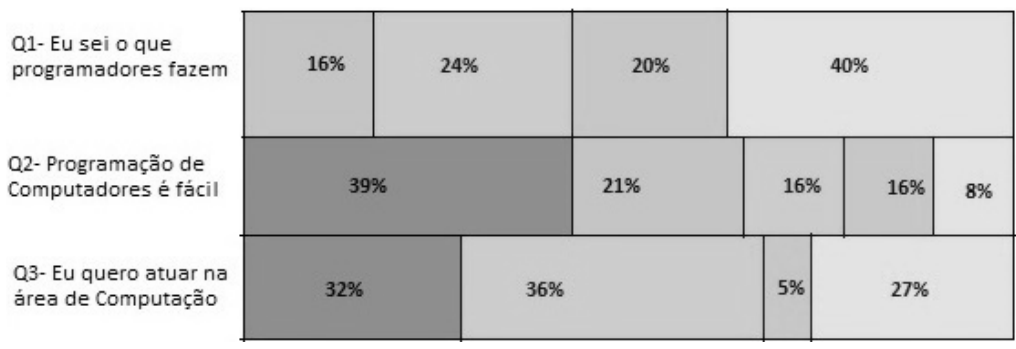

Figura 1 - Questionário Pré-Intervenção

No questionário pós-intervenção foram abordadas questões relacionadas ao desenvolvimento do projeto, percepção dos estudantes sobre os professores e o projeto, além de afirmações semelhantes às apresentadas no questionário pré-intervenção. Com a análise dos dados, foi possível constatar mudanças significativas relacionadas às afirmações presentes no questionário sobre a área da Computação. Assim, cerca de $75 \%$ dos estudantes afirmaram saber o que um profissional de programação faz, demonstrando um crescimento significativo se comparado aos dados obtidos no questionário pré-intervenção. Mesmo com o crescimento em torno da afirmação anterior, os estudantes se mostraram neutros, quanto a afirmação de que a programação de computadores é algo fácil. Em relação a seguir na área da Computação, 59\% concordam que atuar na área de Computação se tornou uma possibilidade, enquanto $23 \%$ se mostraram neutros nesse quesito e, para $17 \%$, atuar na área de Computação não é uma possibilidade. 


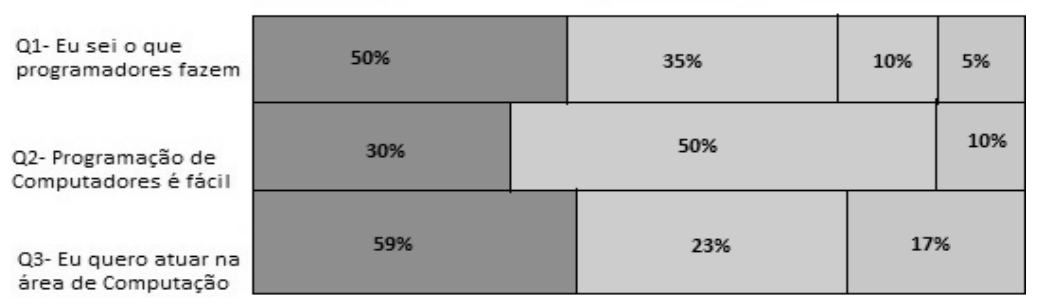

Figura 2 - Questionário Pós-Intervenção

A partir da análise dos dados obtidos dos questionários, foi possível identificar um crescimento significativo da percepção dos estudantes em torno da Computação. Fica claro que a maioria dos estudantes passou a compreender de que forma $o$ profissional de programação pode atuar no mundo do trabalho, além de passar a ver a área como possível ramo de atuação. Para além destes dados, o questionário pós intervenção contou com questões abertas relacionadas às atividades do projeto e metodologia dos professores; como resultado, os estudantes se mostraram satisfeitos com a atuação dos professores, salientando apenas que o tempo de realização das atividades deveria ser maior permitindo a conclusão de mais atividades por dia. Sobre a utilização dos ambientes Hora do Código e App Inventor, para 64\% dos estudantes, a realização das atividades com o App Inventor é mais prazerosa, pois eles conseguem criar aplicativos e visualizar as etapas de construção; por outro lado, para os outros $36 \%$, as atividades com o Hora do Código é mais interessante, devido a interface e as diferentes interações que podem ser feitas com os personagens no processo de solução dos problemas.

\section{Considerações finais}

Conforme exposto nas seções anteriores, faz-se necessário expandir as discussões acerca da presença feminina no ramo da tecnologia, seja na academia, seja dentro dos ambientes de trabalho, em busca da superação de desigualdades historicamente constituídas. Embora o tempo para execução tenha sido curto, para a maioria dos participantes foi por intermédio do projeto aqui apresentado que surgiu a oportunidade de refletir suas realidades de forma crítica, especialmente no tocante às questões de gênero.

Nas atividades finais, percebeu-se que a maioria dos estudantes começaram a construir uma visão mais ampla sobre a disparidade de gênero nos diversos setores da sociedade, e os papéis socialmente construídos e estabelecidos em instituições como a família e a mídia, que contribuem para a internalização da ideia de submissão da mulher ao homem. As rodas de discussão sobre gênero e masculinização na área das ciências exatas foram os momentos em que os participantes apresentaram maior interação entre si, ao expor suas opiniões e vivências cotidianas. As intervenções nesses momentos foram essenciais como pontos de partida; todavia, com o aumento das interações entre os próprios participantes, estes conseguiram estabelecer diálogos que permitiram que as reflexões fossem guiadas pela própria realidade de cada um.

Ficou evidenciado que a inserção das tecnologias no ambiente escolar pode também estimular o desenvolvimento do raciocínio lógico-matemático, a criatividade, a abstração e o Pensamento Computacional. Neste sentido, deve-se destacar a necessidade da base metodológica, sem a qual não seria possível utilizar, de maneira eficaz, as 
VI Congresso Brasileiro de Informática na Educação (CBIE 2017)

Anais dos Workshops do VI Congresso Brasileiro de Informática na Educação (WCBIE 2017)

ferramentas escolhidas para as atividades de programação. Corrobora esta perspectiva as observações sobre o uso inicial e familiarização com o ambiente Hora do Código, quando foi notado que os participantes buscaram meios de solucionar os problemas apresentados por experimentação, via tentativa e erro. Diante deste cenário, foi necessário intervir com provocações aos participantes sobre as resoluções por eles apresentada.

Com este trabalho, promoveu-se o incentivo à participação de grupos pouco representados na área da Computação, evidenciando as vantagens no desenvolvimento de crianças e adolescentes propiciados pela aprendizagem de conceitos da área e a construção do Pensamento Computacional. Por fim, deve-se destacar a importância do conjunto de atividades desenvolvidas no projeto para os participantes, bem como salientar a necessidade de mais iniciativas semelhantes. $\mathrm{O}$ despertar do interesse dos estudantes, em especial, as meninas, pode fazer toda diferença na transformação das suas realidades.

\section{Referências}

Ashcraft, C., Eger, E., \& Friend, M. (2012). Girls in iT: the facts. National Center for Women \& IT. Boulder, CO.

Bell T.C.G., Witten, I. (1995). Computer Science Unplugged: Capturing the interest of the uninterested. Anais do NZ Computer Conference,Wellington, Nova Zelândia.

Bezerra, F., \& Dias, K. (2014). Programação de computadores no ensino fundamental: Experiências com logo e scratch em escola pública. In XXXIV Congresso da SBCXXII Workshop de Ensino de Computação, Brasília.

Cassenote, M. R. S., \& Antoniazzi, R. L. (2015). RELATO DE EXPERIÊNCIA DA UTILIZAÇÃO DA FERRAMENTA LIGHTBOT ENQUANTO CONSTRUTOR DO RACIOCÍNIO PARA LÓGICA DE PROGRAMAÇÃO. Salão do Conhecimento, 1(1).

Cavalcante, A., Costa, L. D. S., \& Araujo, A. L. (2016, November). Um Estudo de Caso Sobre Competências do Pensamento Computacional Desenvolvidas na Programação em Blocos no Code. Org. In Anais dos Workshops do Congresso Brasileiro de Informática na Educação (Vol. 5, No. 1, p. 1117).

CENSO, I. B. G. E. Censo IBGE 2010. Acesso em, v. 23, 2010. Disponível em: << http://www. censo2010. ibge. gov. br/>>.

Creswell, J. W. (2010). Projeto de pesquisa métodos qualitativo, quantitativo e misto. In Projeto de pesquisa métodos qualitativo, quantitativo e misto. Artmed.

CODE, W. M. (2017). Disponível em: <https://www.meetup.com/ptBR/WoMakersCode/>.

Dantas, R. F., \& Costa, F. E. A. D. (2013). CODE: O ensino de linguagens de programação educativas como ferramentas de ensino/aprendizagem. Simpósio Hipertexto e Tecnologias na Educação, 5.

De Souza Martins, H. H. T. (2004). Metodologia qualitativa de pesquisa. Educação e pesquisa, 30(2), 289-300. 
VI Congresso Brasileiro de Informática na Educação (CBIE 2017)

Anais dos Workshops do VI Congresso Brasileiro de Informática na Educação (WCBIE 2017)

Faria, E. T. (2004). O professor e as novas tecnologias. Ser professor, 4, 57-72.

França, R., \& Tedesco, P. (2015, October). Desafios e oportunidades ao ensino do Pensamento Computacional na educação básica no Brasil. In Anais dos Workshops do Congresso Brasileiro de Informática na Educação (Vol. 4, No. 1, p. 1464).

Gomes, T. C., \& de Melo, J. C. (2013). App Inventor for Android: Uma nova possibilidade para o ensino de lógica de programação. In Anais dos Workshops do Congresso Brasileiro de Informática na Educação (Vol. 2, No. 1).

Gomes, T., \& Melo, J. (2013). O Pensamento Computacional no Ensino Médio: Uma Abordagem Blended-Learning. In Anais do XXI Workshop sobre Educação em Computação-XXXIII Congresso da Sociedade Brasileira de Computação. Maceió, AL-Brasil.

MIT - Massachussets Institute of Technology (2012). “App Inventor for Android”, appinventor.mit.edu, Agosto.

Pinto, A. M. (2004). As novas tecnologias e a educação. ANPED SUL, 6, 1-7.

Wing, J. M. (2006). Computational thinking. Communications of the ACM, 49(3), 3335 .

Wing, J. (2016). Pensamento Computacional-Um conjunto de atitudes e habilidades que todos, não só cientistas da computação, ficaram ansiosos para aprender e usar. Revista Brasileira de Ensino de Ciência e Tecnologia, 9(2). 